\title{
Autenticidad enunciativa \\ y sociosemántica del pop-rock en español
}

\author{
María MARTíNEZ CASAS (Eichstätt)
}

\section{Summary}

From a sociological perspective, the aesthetic value of authenticity is considered a key feature musicians must have in order to enter the pop-rock canon (Val, Noya \& Pérez-Colman 2014; Appen $\&$ Doehring 2006). The present paper aims to identify and analyse different strategies employed to suggest authenticity regarding enunciation (Laferl 2005) and social semiotics (Halliday 1978) in pop-rock song lyrics. To this end, a 120.000-word corpus of 722 songs in Spanish was compiled and analysed in terms of frequencies, participants, key words and practice patterns.

\section{Introducción}

Partiendo de las tesis de Pierre Bourdieu sobre sociología del arte así como de la obra de Motti Regev acerca de la indigenización del rock, los sociólogos españoles Val, Noya y PérezColman publicaron en 2014 un canon estético del pop-rock español basado en el reconocimiento de la crítica y de las instituciones de consagración nacional de dicho género musical. En la lista jerárquica de 50 artistas que resultó de su estudio, los autores constataron dos características determinantes de los músicos más valorados: la hibridación y la autenticidad. Por una parte, la hibridación surge de la traducción cultural de un género musical procedente del ámbito anglosajón y resulta en una expresión de diferenciación local:

La hibridación, mestizaje o indigenización se justifica como autenticidad local, o singularidad nacional, y se puede observar empíricamente en que se canta en lengua vernácula, se habla sobre cuestiones locales (históricas, políticas, sociales, etc.), mezcla de la metrópoli con el folclore nacional y la utilización de instrumentos locales (Val, Noya y Pérez-Colman 2014, 153, cursivas en original).

Por otra parte, la autenticidad referida a los artistas se expresa en su capacidad de "crear un personaje creíble cada vez que se suben a un escenario" (Val, Noya y Pérez-Colman 
2014, 158) y remite de este modo a la puesta en escena de las canciones. Se trata de una autenticidad en el plano performativo que Appen y Doehring (2006) también destacan en su canon del pop-rock anglosajón, en el que ofrecen una lista de los 30 álbumes de pop-rock anglosajón más valorados desde una perspectiva sociológica y estética que sirve de precedente del canon de los sociólogos españoles. Para Appen y Dohering, "Musicians who do not create an impression of making music that matters personally to them have no chance to be successful in the lists we document" (Appen y Dohering 2006, 31). Se plantea por tanto la pregunta de qué mecanismos poseen los músicos para articular esa autenticidad en relación con las canciones que componen, escriben (texto) y/o interpretan (puesta en escena).

Entre los numerosos estudios que han tematizado la construcción de autenticidad en el plano performativo de la música popular (entre otros, Frith 1989 y 1996, Auslander 2006, Moore 2002, Eckstein 2010, Brackett 2008), Appen (2013, 41-44), categoriza cuatro dimensiones de autenticidad (personal, sociocultural, artesanal y emocional) y se centra en analizar mecanismos concretos para su construcción en la puesta en escena de canciones de pop-rock anglosajón.

Más allá de las estrategias asociadas a la autenticidad en el plano performativo que abordan dichos autores (la voz, los personajes musicales, el proceso de autentificación, etc.), el objetivo del presente artículo es considerar el nivel enunciativo de las letras de canciones de pop-rock para iluminar los mecanismos de los que se sirven los artistas a nivel textual con el objetivo de satisfacer las convenciones y expectativas relacionadas con la autenticidad en el pop-rock. A fin de identificar patrones enunciativos y sociosemánticos se presenta en primer lugar un corpus compilado en base al canon estético del pop-rock español de Val, Noya y Pérez-Colman que a continuación será analizado cuantitativa y cualitativamente.

\section{Hacia un corpus de pop-rock en español}

Como se ha mencionado en la introducción, el canon estético del pop-rock en español está formado por una lista jerárquica de 50 artistas y sus álbumes y/o canciones más valorados (Val, Noya y Pérez-Colman 2014, 165-167). En un primer estadio, para la construcción del corpus se tuvo en cuenta el álbum con mayor puntuación de cada artista. De este modo, se logró compilar un total de 350 letras de canciones (54.688 palabras) correspondientes a 29 álbumes editados entre 1968 y 2007 (Apéndice 1).

Sin embargo, dado que el canon está compuesto mayoritariamente por álbumes editados en la década de los años 80 por artistas masculinos españoles (unas características que, en términos sociológicos, reflejan las narraciones hegemónicas surgidas de las disposiciones comunes de los críticos que valoraron a los artistas, cf. Fouce 2013,73), resultaba necesario ampliar el corpus para alcanzar una mayor diversidad en cuanto a épocas, nacionalidades y género. Siguiendo los mecanismos de consagración que mencionan Val, Noya y PérezColman en su canon $(2014,151)$, se optó por aumentar el corpus con los álbumes de artistas galardonados por la Academia Latina de Artes y Ciencias de la Grabación con un Grammy 
latino (en cuanto reconocimiento profesional mediante dicha legitimación específica). Con ello, en un segundo estadio se compilaron 372 letras de canciones (69.222 palabras) procedentes de 35 álbumes galardonados entre 1987 y 2015 (Apéndice 2).

Por lo tanto, el corpus de letras de pop-rock en español que sirve de base para el presente análisis consta en la actualidad (junio de 2016) de 722 letras de canciones escritas en español (123.910 palabras) correspondientes a 64 álbumes editados entre 1968 y 2015 por 61 solistas o grupos de pop-rock procedentes (en orden de representatividad) de España, México, Colombia, Argentina, Puerto Rico, Chile, Uruguay, República Dominicana, Estados Unidos, Italia y Canadá. Las letras que componen el corpus han sido transcritas de 50 álbumes (cedés, discos, casetes) y un libro (dos álbumes); en el caso de 13 álbumes se han extraído de la página electrónica oficial de los artistas. Todas las canciones se encuentran archivadas en formato digital para su posterior análisis informático con los programas WordSmith Tools 6.0 y AntConc 3.4.4w.

\section{Autenticidad enunciativa}

La lista de las 20 formas (types) más frecuentes del corpus de pop-rock en español apunta al carácter enunciativo mayoritariamente (inter-)personal a través de los principales participantes de las canciones analizadas, a saber, el "yo" (me, mi, yo) y el "tú" (te, tu), señalados en negrita en la siguiente tabla:

Tabla 1. Lista de 20 formas más frecuentes del corpus de pop-rock en español.

\begin{tabular}{|l|l|l|l|l|l|}
\hline $\mathrm{N}$ & Word & Freq. & $\%$ & Texts & $\%$ \\
\hline 1 & QUE & 4.902 & 3,95 & 667 & 92,38 \\
\hline 2 & DE & 4.062 & 3,27 & 673 & 93,21 \\
\hline 3 & Y & 3.981 & 3,21 & 668 & 92,52 \\
\hline 4 & NO & 3.203 & 2,58 & 598 & 82,83 \\
\hline 5 & LA & 3.123 & 2,52 & 629 & 87,12 \\
\hline 6 & EL & 2.799 & 2,26 & 633 & 87,67 \\
\hline 7 & A & 2.669 & 2,15 & 595 & 82,41 \\
\hline $\mathbf{8}$ & ME & $\mathbf{2 . 3 5 7}$ & $\mathbf{1 , 9 0}$ & $\mathbf{5 2 7}$ & $\mathbf{7 2 , 9 9}$ \\
\hline 9 & EN & 2.297 & 1,85 & 624 & 86,43 \\
\hline 10 & UN & 1.666 & 1,34 & 527 & 72,99 \\
\hline $\mathbf{1 1}$ & TE & $\mathbf{1 . 6 4 1}$ & $\mathbf{1 , 3 2}$ & $\mathbf{4 1 9}$ & $\mathbf{5 8 , 0 3}$ \\
\hline $\mathbf{1 2}$ & MI & $\mathbf{1 . 4 7 3}$ & $\mathbf{1 , 1 9}$ & $\mathbf{4 3 3}$ & $\mathbf{5 9 , 9 7}$ \\
\hline
\end{tabular}




\begin{tabular}{|l|l|l|l|l|l|}
\hline 13 & ES & 1.222 & 0,99 & 417 & 57,76 \\
\hline 14 & LO & 1.139 & 0,92 & 394 & 54,57 \\
\hline $\mathbf{1 5}$ & YO & $\mathbf{1 . 0 8 5}$ & $\mathbf{0 , 8 7}$ & $\mathbf{3 7 5}$ & $\mathbf{5 1 , 9 4}$ \\
\hline 16 & POR & 1.074 & 0,87 & 427 & 59,14 \\
\hline 17 & SE & 1.026 & 0,83 & 388 & 53,74 \\
\hline $\mathbf{1 8}$ & TU & $\mathbf{1 . 0 1 9}$ & $\mathbf{0 , 8 2}$ & $\mathbf{3 3 6}$ & $\mathbf{4 6 , 5 4}$ \\
\hline 19 & LOS & 914 & 0,74 & 352 & 48,75 \\
\hline 20 & SI & 855 & 0,69 & 323 & 44,74 \\
\hline
\end{tabular}

Según los resultados que se recogen en la Tabla 1, el "yo" aparece en un mínimo del 73\% de las canciones analizadas y el "tú", en un 58\%. Por tanto, lingüísticamente se constata el uso significativamente frecuente de expresiones deícticas (pronombres personales, determinantes posesivos) de primera y segunda persona del singular como un mecanismo de construcción de autenticidad personal (Appen 2013, 42). A este respecto, los estudios existentes de corpus de pop-rock en inglés también han resaltado el estilo conversacional y el interés humano que caracterizan las canciones de pop-rock (Murphey 1990) mediante el uso de expresiones autorreferenciales (Werner 2012) que apuntan a una producción involucrada (Bértoli-Dutra 2014).

Adaptando métodos de análisis de discurso y de estudios literarios, Laferl (2005, 68-69) propone categorizar la estructura enunciativa de las canciones de música popular en función de la presencia o ausencia de un "yo" articulado en las letras. Según un esquema adaptado de su propuesta, el corpus de pop-rock en español arroja los siguientes resultados:

Tabla 2. Esquema adaptado de Laferl 2005 y resultados del corpus de pop-rock en español.

\begin{tabular}{|c|c|c|c|c|c|}
\hline A. & Canciones con un "yo" articulado: & & & 646 & $89,47 \%$ \\
\hline A.1. & el "yo" habla sobre sí mismo; & 158 & $21,88 \%$ & & \\
\hline A.2. & $\begin{array}{l}\text { el "yo" habla sobre otra persona o cuenta una } \\
\text { historia ajena; }\end{array}$ & 27 & $3,73 \%$ & & \\
\hline A.3. & $\begin{array}{l}\text { el "yo" se dirige a un "túc" y habla sobre su rel- } \\
\text { ación común; }\end{array}$ & 401 & $55,54 \%$ & & \\
\hline A. 4 . & el "yo" explica al "tú" una historia ajena. & 60 & $8,31 \%$ & & \\
\hline B. & Canciones sin un "yo" articulado: & & & 76 & $10,52 \%$ \\
\hline B.1. & canciones narrativas; & 69 & $9,55 \%$ & & \\
\hline B.2. & diálogos. & 7 & $0,96 \%$ & & \\
\hline Total: & & 722 & $100 \%$ & 722 & $100 \%$ \\
\hline
\end{tabular}


A nivel enunciativo, por tanto, se matizan al alza los resultados cuantitativos obtenidos en la lista de formas más frecuentes: alrededor del $89 \%$ de las letras analizadas incluyen un "yo" articulado (A) y este se dirige a un "tú" (quien participa en un 64\% de letras) para hablar sobre su relación común (A.3). Dicha estructura más frecuente ya se introduce como marco enunciativo en el primer verso de un gran número de canciones, como se ilustra en los siguientes ejemplos:

1. "Tu voz me hace sentir como en un sueño" ("De verdad", Alejandra Guzmán)

2. "Si tú vinieras a cenar esta noche conmigo" ("La canción del pescado", Álex, Jorge y Lena)

3. "Tu rostro lo tengo fijado" ("El álbum”, Aterciopelados)

La relación común de la que el "yo" habla al "tư" tematiza en contadas ocasiones la amistad (ejemplo 4) o el parentesco (ejemplo 5) y trata en su gran mayoría de un vínculo de índole amorosa (amor y/o desamor) o sexual (ejemplos 6-9):

4. "Tú eres mi hermano del alma, realmente el amigo" ("Amigo", Marc Anthony)

5. "Hola viejo dime cómo estás" ("Quizás", Enrique Iglesias)

6. "han pasado tres años desde aquel verano y sigo enamorado de ti" ("El bello verano", Family)

7. "Cómo pudiste hacerme estoy a mí / yo que te hubiese querido hasta el fin" (“Cómo pudiste hacerme esto a mí”, Alaska y Dinarama)

8. "Como garfios de plata se clavan tus dedos en mi cuerpo y tu risa se gasta entre mis besos encendidos" ("Los ángeles no tienen hélices", El Último de la Fila)

9. "Nos fundiremos / los dos en un cuerpo" ("La reclusa", Golpes bajos)

Cabe mencionar por último que los resultados obtenidos por Laferl en su estudio sobre canciones de otras décadas (de 1920 a 1960), otros países (Brasil, Cuba, Martinica y Trinidad), otros géneros (en concreto: samba, marcha, bolero, son, beguine y calypso) y, en parte, otras lenguas (portugués, francés) arrojan porcentajes similares en cuanto a la estructura enunciativa y temática predominantes en las letras de música popular (Laferl 2005, 72).

\section{¿Pop de dos y rock de uno?}

En relación con el presente análisis, no obstante, conviene matizar el concepto de pop-rock, usado hasta el momento de modo grueso según la nomenclatura de la crítica especializada (canon estético) y de las instituciones de consagración (Academia Latina de Artes y Ciencias de la Grabación). Con el objetivo de iluminar posibles diferencias enunciativas, se aconseja un análisis diferenciado de los subgéneros musicales que recoge el presente corpus. 
A tal efecto cabe tener en cuenta el hecho de que, por una parte, los sociólogos españoles distinguen en su canon estético los siguientes ocho géneros y escenas: movida, rock, rock urbano, flamenco, pop, indie, cantautores y folk-rock. Por otra parte, la Academia Latina de Artes y Ciencias de la Grabación concede los premios Grammy latinos en 19 áreas, de las que resultan relevantes para el presente corpus las siguientes categorías: pop, rock, pop-rock y grabación del año (Tabla 3). Los géneros musicales de los únicos tres artistas que forman parte tanto del canon estético como de la lista de galardonados con un premio Grammy latino (Alejandro Sanz, Andrés Calamaro y Miguel Bosé) coinciden en la clasificación de ambas listas (Sanz y Bosé, pop; Calamaro, rock), por lo que se demuestra cierta estabilidad en la asignación de subgéneros musicales a artistas (Apéndices 1 y 2).

Tabla 3. Composición del corpus según subgéneros musicales.

\begin{tabular}{|l|l|l|l|l|l|l|l|l|l|l|}
\hline & Movida & Rock & $\begin{array}{l}\text { Rock } \\
\text { urbano }\end{array}$ & Flamenco & Pop & Indie & $\begin{array}{l}\text { Cantau- } \\
\text { tor }\end{array}$ & $\begin{array}{l}\text { Folk- } \\
\text { rock }\end{array}$ & $\begin{array}{l}\text { Pop- } \\
\text { rock }\end{array}$ & Total \\
\hline Letras & 72 & $\mathbf{2 0 9}$ & 13 & 40 & $\mathbf{2 6 4}$ & 14 & 77 & 11 & 22 & 722 \\
\hline$\%$ & 9,97 & $\mathbf{2 8 , 9 4}$ & 1,80 & 5,54 & $\mathbf{3 6 , 5 6}$ & 1,93 & 10,66 & 1,52 & 3,04 & 100 \\
\hline
\end{tabular}

Atendiendo a dicha clasificación y visto que los dos subgéneros más representados en el corpus son el pop con 264 canciones y el rock con 233 canciones (cifra que resulta de la suma de rock, rock urbano y folk-rock), se muestran a continuación los resultados del análisis de la estructura enunciativa de las letras de álbumes cuyos artistas se adscriben a los géneros musicales de ambos subgéneros:

- Las canciones correspondientes a álbumes considerados pop ascienden a 264, un $36,56 \%$ del total del corpus. La estructura enunciativa A3 (el "yo" se dirige a un "tú" y habla sobre su relación común) se registra en un $82,50 \%$ de estas canciones, mientras que la estructura A1 (el "yo" habla sobre sí mismo) alcanza un 10,26\%. Respecto a los resultados generales obtenidos en el análisis del corpus (Tabla 2) destaca la marcada preferencia por la estructura enunciativa A3, especialmente en detrimento de la estructura A1.

- Las canciones correspondientes a álbumes considerados rock (rock, rock urbano y folk-rock) ascienden a 233 , un $32,27 \%$ del total del corpus. Al contrario que en el caso del pop, la estructura enunciativa A3 desciende sustancialmente a un $45,06 \%$ en este subcorpus, mientras que la estructura A1 (el "yo" habla sobre sí mismo) alcanza un significativo 28,75\%, la estructura B1 (canciones narrativas) registra un 12,01\% y la estructura A4 (el "yo" explica al "tú" una historia ajena) se encuentra en un 9,01\% de las letras. 
El análisis de la estructura enunciativa de las letras de canciones pop-rock en función del subgénero musical que se adscribe a los artistas correspondientes cristaliza el mecanismo de construcción de autenticidad del pop-rock en una base lírica, pero con matizaciones en función del subgénero musical, como puntualiza Jaedtke $(2000,207)$ partiendo de un concepto amplio de "música pop" (entendida como música popular):

Alle Popmusik, egal welcher Richtung, ist primär Ausdrucksmusik und auf das Individuum als fühlendes Subjekt bezogen [...]. Varianten davon sind Artikulationen gesellschaftlicher Verhältnisse (bezogen auf Gruppen von Menschen) wie schon früher in Rock- und Beatmusik und neuerdings in Formen des Rap. Grundsätzlich aber ist die Haltung von Popmusik eine lyrische: nicht erzählende, nicht dramatisierend.

Del análisis de las palabras clave de cada subgénero musical en relación con el corpus total (de referencia) se desprende también dicha matización enunciativa, cuya distribución (range, es decir, el número de letras en las que aparece) también se indica en la siguiente tabla:

Tabla 4. Palabras clave del subcorpus de pop.

\begin{tabular}{|l|l|l|l|l|}
\hline Rank. & Freq. & Keyness & Keyword & Range \\
\hline $\mathbf{1}$ & $\mathbf{3 5 3}$ & $\mathbf{5 2 . 5 4 2}$ & $\mathbf{t i}$ & $\mathbf{1 1 2}$ \\
\hline 2 & 2368 & 43.521 & que & --- \\
\hline 3 & 107 & 39.380 & coro & --- \\
\hline $\mathbf{4}$ & $\mathbf{5 4 9}$ & $\mathbf{2 6 . 3 5 2}$ & tu & $\mathbf{1 4 2}$ \\
\hline $\mathbf{5}$ & $\mathbf{1 0 7}$ & $\mathbf{2 5 . 0 6 7}$ & contigo & $\mathbf{4 5}$ \\
\hline $\mathbf{6}$ & $\mathbf{2 2 1}$ & $\mathbf{2 4 . 0 1 3}$ & mí & $\mathbf{9 3}$ \\
\hline 7 & $\mathbf{4 1 9}$ & $\mathbf{2 1 . 8 6 7}$ & tú & $\mathbf{1 2 8}$ \\
\hline 8 & 127 & 20.118 & cómo & --- \\
\hline $\mathbf{9}$ & $\mathbf{8 1}$ & $\mathbf{1 9 . 0 7 1}$ & mía & $\mathbf{1 6}$ \\
\hline 10 & 54 & 18.254 & marcha & 3 \\
\hline
\end{tabular}

De manera consistente con el análisis de la estructura enunciativa de las canciones de artistas adscritos al pop, cobran aquí mayor importancia las palabras clave relacionadas con los deícticos de segunda persona del singular (ti, tu, contigo, tú) y ligeramente los de primera persona del singular (mí, mía), que apuntan a la construcción de una autenticidad emocional y personal (Appen 2013, 42-44): 
1. "Sabes bien que sólo te quiero a ti” (“One, two, three GO!", Belanova)

2. "Te mentiría si negara hoy / que desde entonces sólo sueño / contigo" "Apareces tú", La oreja de Van Gogh)

3. "Ahora que tú y yo somos de verdad" ("Búscame", Rosario)

4. "Pero, perdona, amiga mía, / no es inteligencia, ni es sabiduría; / esta es mi manera de decir las cosas. / No es que sea mi trabajo, es que es mi idioma" "Amiga mía", Alejandro Sanz)

En este último ejemplo citado de una letra escrita por Alejandro Sanz se destaca el énfasis de la autenticidad artesanal (Appen 2013, 43), vinculada de modo estrecho con la autenticidad personal: escribir o cantar canciones no consiste para él meramente en su "trabajo", sino en su "idioma", es decir, su manera de comunicarse.

Otro tipo de autenticidad personal se ve reflejada en las letras de artistas considerados rock, en las que destaca precisamente la palabra clave "rock", que apunta al discurso sobre el propio género musical combinado con la estructura enunciativa centrada en el "yo":

Tabla 5. Palabras clave del subcorpus de rock.

\begin{tabular}{|l|l|l|l|l|}
\hline Rank. & Freq. & Keyness & Keyword & Range \\
\hline $\mathbf{1}$ & $\mathbf{4 8}$ & $\mathbf{2 9 . 8 8 5}$ & rock & $\mathbf{1 9}$ \\
\hline 2 & 32 & 21.215 & bis & --- \\
\hline 3 & 36 & 20.424 & estribillo & --- \\
\hline 4 & 25 & 17.678 & dragón & 1 \\
\hline 5 & 27 & 17.530 & paula & 2 \\
\hline 6 & 33 & 15.710 & veneno & 6 \\
\hline 7 & 48 & 15.367 & uno & --- \\
\hline 8 & 35 & 13.738 & doy & 9 \\
\hline 9 & 38 & 13.222 & dormir & 10 \\
\hline 10 & 19 & 12.423 & sucede & 2 \\
\hline
\end{tabular}

De este modo, la autenticidad se consagra a nivel textual mediante el metadiscurso sobre el género musical que se representa (ejemplos 14-15) en cuanto autenticidad sociocultural (Appen 2013, 43), por lo que se podría hablar más bien de un rock de "muchos", pero sin renunciar a los mecanismos de construcción de autenticidad personal y emocional (ejemplos 16-17) propios de la música popular: 
1. “y por mi afición suicida preferida / rock de verdad" ("No tan Buenos Aires”, Andrés Calamaro)

2. "Ayúdanos a conectar / sólo por ti el rock existirá” ("Bienvenidos”, Miguel Ríos)

3. "Yo soy así / no hay nada que pueda cambiar / un sentimiento sin igual / desde que el mundo está girando" ("Esclavo de tu amor", Vicentico)

4. "Y mírame y verás que mis ojos te aman más / Y mírame y verás que te digo la verdad" ("La única”, Juanes)

\section{Autenticidad sociosemántica}

Partiendo de los resultados obtenidos en el análisis de la estructura enunciativa, por los que el mecanismo más frecuente de construcción de autenticidad estriba en la articulación del "yo" o bien del "yo" y del "tú", cabe preguntarse qué acciones llevan a cabo cada uno de los participantes y cómo se construye la autenticidad en base a dichas acciones. Para ello se propone un análisis de los patrones lingüísticos más frecuentes con deícticos de primera y segunda persona del singular (Tabla 1) según los procesos sociosemánticos postulados por Halliday 1978 (en concreto: procesos materiales, mentales, existenciales, relacionales, verbales y conductuales).

Los patrones de uso lingüístico más frecuentes (cf. Bubenhofer 2009) que se registran con "me" en cuanto pronombre reflexivo (Tabla 6) y pronombre de objeto (Tabla 7) iluminan diferencias sustanciales entre los planos sociosemánticos en los que se sitúan las acciones que llevan a cabo el "yo" y el "tú" (ordenadas aquí según su distribución o range):

Tabla 6. Patrones de actuación del "yo" con verbos reflexivos.

\begin{tabular}{|l|l|l|l|}
\hline Rank & Freq & Range & Cluster \\
\hline $\mathbf{1}$ & $\mathbf{3 4}$ & $\mathbf{1 9}$ & me siento \\
\hline $\mathbf{2}$ & $\mathbf{1 7}$ & $\mathbf{1 3}$ & me quedo \\
\hline $\mathbf{3}$ & $\mathbf{1 7}$ & $\mathbf{1 2}$ & me vuelvo \\
\hline 4 & 21 & 10 & me muero \\
\hline 5 & 20 & 10 & me puedo \\
\hline $\mathbf{6}$ & $\mathbf{1 1}$ & 7 & me pongo \\
\hline 7 & 7 & 7 & me pregunto \\
\hline 8 & 10 & 7 & me quiero \\
\hline $\mathbf{9}$ & $\mathbf{2 4}$ & $\mathbf{6}$ & me acuerdo \\
\hline 10 & 6 & 6 & me encuentro \\
\hline
\end{tabular}


Tabla 7. Patrones de interacción "tú" "a mí" en presente de indicativo, subjuntivo y en imperativo.

\begin{tabular}{|l|l|l|l|l|l|l|l|}
\hline Rank & Freq & Range & Cluster & Rank & Freq & Range & Cluster \\
\hline $\mathbf{1}$ & $\mathbf{2 0}$ & $\mathbf{1 2}$ & me das & $\mathbf{1}$ & $\mathbf{8 8}$ & $\mathbf{4 0}$ & dime \\
\hline 2 & 12 & 9 & me ves & $\mathbf{2}$ & $\mathbf{5 0}$ & $\mathbf{1 8}$ & dame \\
\hline $\mathbf{3}$ & $\mathbf{1 2}$ & $\mathbf{7}$ & me dejes & $\mathbf{3}$ & $\mathbf{3 8}$ & $\mathbf{1 8}$ & déjame \\
\hline $\mathbf{4}$ & $\mathbf{8}$ & $\mathbf{7}$ & me digas & 4 & 29 & 12 & mírame \\
\hline 5 & 8 & 7 & me has & $\mathbf{5}$ & $\mathbf{1 1}$ & $\mathbf{8}$ & llévame \\
\hline $\mathbf{6}$ & $\mathbf{9}$ & $\mathbf{6}$ & me pidas & $\mathbf{6}$ & $\mathbf{7}$ & $\mathbf{4}$ & abrázame \\
\hline 7 & 7 & 6 & me puedes & 7 & 5 & 4 & escúchame \\
\hline 8 & 9 & 6 & me quieres & 8 & 6 & 4 & perdóname \\
\hline $\mathbf{9}$ & $\mathbf{6}$ & $\mathbf{5}$ & me dices & $\mathbf{9}$ & $\mathbf{1 9}$ & $\mathbf{3}$ & bésame \\
\hline 10 & 6 & 5 & me tienes & 10 & 7 & 3 & óyeme \\
\hline
\end{tabular}

Mientras que el "yo" lleva a cabo acciones que se pueden adscribir principalmente a un plano mental (sentirse; quedarse, volverse y ponerse como verbos de cambio, acordarse, encontrarse), el "tú" se sitúa en un plano material (dar, dejar, llevar, abrazar, besar) y verbal (decir, pedir). De ahí se puede postular que el patrón discursivo más frecuente de las canciones de pop-rock analizadas responde a la siguiente estructura: el "tú" hace algo (proceso material) que sume al "yo" en un estado reflexivo (proceso mental). La autenticidad textual se construye de este modo mediante la expresión de los procesos mentales que lleva a cabo el "yo" y la apelación retórica al "tư" a través de las frecuentes formas de imperativo.

Dichas tendencias sociosemánticas se ven confirmadas en los resultados que recogen las acciones más frecuentes vinculadas a la forma "te" en cuanto pronombre reflexivo (Tabla 8) y pronombre de objeto (Tabla 9):

Tabla 8. Patrones de actuación del "tú" con pronombres reflexivos.

\begin{tabular}{|l|l|l|l|l|l|l|l|}
\hline Rank & Freq & Range & Cluster & Rank & Freq & Range & Cluster \\
\hline $\mathbf{1}$ & $\mathbf{5 1}$ & $\mathbf{2 7}$ & te vas & $\mathbf{1}$ & $\mathbf{1 8}$ & $\mathbf{1 1}$ & vete \\
\hline 2 & 9 & 8 & te has & 2 & 16 & 10 & quédate \\
\hline $\mathbf{3}$ & 7 & $\mathbf{6}$ & te vayas & 3 & 9 & 6 & déjate \\
\hline 4 & 7 & 5 & te acuerdas & $\mathbf{4}$ & $\mathbf{1 7}$ & $\mathbf{5}$ & llévate \\
\hline 5 & 9 & 5 & te quedes & $\mathbf{5}$ & $\mathbf{4}$ & $\mathbf{4}$ & acércate \\
\hline
\end{tabular}




\begin{tabular}{|l|l|l|l|l|l|l|l|}
\hline 6 & 8 & 4 & te das & $\mathbf{6}$ & $\mathbf{3}$ & $\mathbf{3}$ & entrégate \\
\hline 7 & $\mathbf{4}$ & $\mathbf{4}$ & te marchas & 7 & $\mathbf{2}$ & $\mathbf{2}$ & búscate \\
\hline $\mathbf{8}$ & $\mathbf{3}$ & $\mathbf{3}$ & te acercas & 8 & 3 & 2 & estate \\
\hline $\mathbf{9}$ & $\mathbf{4}$ & $\mathbf{3}$ & te fueras & $\mathbf{9}$ & $\mathbf{2}$ & $\mathbf{2}$ & levántate \\
\hline 10 & 10 & 3 & te olvides & 10 & 4 & 2 & mírate \\
\hline
\end{tabular}

Tabla 9. Patrones de interacción "yo" "a ti" en presente de indicativo.

\begin{tabular}{|l|l|l|l|}
\hline Rank & Freq & Range & Cluster \\
\hline $\mathbf{1}$ & $\mathbf{1 0 8}$ & $\mathbf{4 7}$ & te quiero \\
\hline 2 & 41 & 18 & te digo \\
\hline 3 & 27 & 15 & te puedo \\
\hline $\mathbf{4}$ & $\mathbf{1 8}$ & $\mathbf{1 2}$ & te amo \\
\hline 5 & 14 & 11 & te busco \\
\hline 6 & 12 & 10 & te pido \\
\hline 7 & $\mathbf{1 0}$ & $\mathbf{9}$ & te veo \\
\hline 8 & 15 & 8 & te espero \\
\hline $\mathbf{9}$ & $\mathbf{1 1}$ & $\mathbf{8}$ & te miro \\
\hline 10 & 15 & 8 & te tengo \\
\hline
\end{tabular}

En este caso, las acciones más frecuentes que lleva a cabo el "tú" continúan situándose en un plano material y pertenecen en su mayor parte a verbos de movimiento (irse, marcharse, acercarse, levantarse). En cuanto a las acciones protagonizadas por el "yo", se confirma su valor sociosemántico mental con verbos como querer, amar, ver, mirar y otros resultados como extrañar, sentir, conocer, necesitar, olvidar, recordar, soñar. Destacan por último los patrones "te digo" y "te pido" como procesos verbales, si bien se sitúan en frecuencia (53) por detrás de las colocaciones protagonizadas por el "tú" (102).

A nivel sociosemántico se puede concluir que el "yo" se representa en las letras de poprock como un participante sumido en un plano mental provocado por los procesos materiales que lleva a cabo el "tú" (ejemplos 18-20):

1. "El verano que estuviste allá en la playa / y yo estaba solo en casa / sin saber lo que pasaba / tú no me llamaste ni una sola vez" ("La playa”, Los Planetas)

2. "Ay, amor me duele tanto / que te fueras sin decir adónde” ("La tortura”, Shakira) 
3. "Déjame, que yo no tengo la culpa de verte caer" ("Entre dos tierras", Héroes del silencio)

Dicho patrón de interacción entre el "yo" y el "tú" puede incluso llevar a que el "yo" se exprese a merced del "tú" (ejemplo 21) o dependa de las acciones materiales del mismo para poder llevar a cabo otros procesos no mentales (ejemplos 22-23):

4. "Dime pa' dónde vas con mi vida" ("Pasos de gigante", Bacilos)

5. "Luna, luna, luna llena / Toma en tu mano, la mía / Llévame a donde te canta, / Mirándote, Simón Díaz" ("Luna de Rasquí", Jorge Drexler)

6. "Sácame algún día del corral: necesito salir" ("Sucede”, Extremoduro)

\section{Resumen y proyección}

El análisis lingüístico (frecuencias, palabras clave), enunciativo (presencia o ausencia de "yo" articulado) y sociosemántico (procesos de los principales participantes) del presente corpus de pop-rock en español, compuesto por 722 letras de canciones y compilado según criterios sociológicos, señala los siguientes tres mecanismos de construcción de autenticidad a nivel lingüístico y discursivo: en primer lugar, entre la lista de formas más frecuentes destacan los deícticos de primera y segunda personal del singular; en segundo lugar, la mayor parte de letras analizadas contienen un "yo" articulado, que se dirige a un "tú" para hablarle de su relación común, siendo esta de índole amorosa; por último, los patrones sociosemánticos más frecuentes del "yo" se sitúan en un plano mental, mientras que las acciones que lleva a cabo el "tú" corresponden predominantemente a procesos materiales y verbales.

Por tanto se puede postular que los mecanismos para construir autenticidad a nivel textual se basan fundamentalmente en la enunciación a través de un "yo" que habla al "tú" sobre lo que siente, piensa y percibe (autenticidad personal y emocional).

Los resultados similares obtenidos en algunos corpus de pop-rock en inglés (comentario de la Tabla 1) así como en un corpus de otros géneros musicales en varias lenguas románicas (comentario de la Tabla 2) apuntan a una posible preferencia en la construcción de autenticidad a nivel textual que sería común a un amplio número de manifestaciones de música popular en culturas occidentales. Un estudio comparativo sobre los patrones de uso lingüístico en varias lenguas podría corroborar o desmentir hasta qué punto se reflejan esas similitudes a nivel enunciativo.

Estrechamente ligado con este aspecto, se plantea la cuestión de la construcción de la hibridación como autenticidad local. El presente estudio solo ha constatado la marcada preferencia por la temática amorosa en las letras analizadas (de modo que, en términos de frecuencia, solo se hablaría marginalmente sobre cuestiones locales). No obstante, en el subgénero del rock, y enmarcados en las estrategias de construcción de autenticidad sociocultural, se pueden encontrar varios ejemplos de hibridación cultural: 
1. "Y ahora estoy aquí sentado / en un viejo Cadillac de segunda mano (...) / aquí en la ladera del Tibidabo" ("Cadillac solitario", Loquillo y Trogloditas)

2. "Vamos a tocar un rock and roll a la plaza del pueblo" ("Rock and roll en la plaza del pueblo" Tequila, en el corpus como versión de Miguel Ríos)

Como se observa en estos ejemplos, los símbolos "Cadillac", "segunda mano" y "rock and roll”, posibles referentes de la cultura estadounidense (Elvis Presley, un coche de segunda mano, surgimiento del género musical), se sitúan en escenarios locales (“Tibidabo" y "plaza del pueblo"). Analizar sistemáticamente este tipo de ejemplos podría iluminar hasta qué punto y de qué manera se articula la hibridación en las letras de pop-rock en español, particularmente en subgéneros musicales como el rock y el flamenco.

Por último, existe un aspecto obviado en este estudio que, sin embargo, resulta fundamental para la construcción de la autenticidad enunciativa basada en un "yo" y un "tú": el género. A través del análisis de sus marcas en el texto (ya sean gramaticales o sociales, cf. Laferl 2005, 74-83), por ejemplo, mediante el desplazamiento enunciativo del "yo" y el "tú" al "él” y "ella" o mediante los patrones de uso lingüístico que se detectan con los pronombres posesivos de primera y segunda persona del singular ("mis ojos", "mis sueños", "mis amigos"; "tus ojos", "tus besos”, "tus labios”) se podría iluminar qué mecanismos de autenticidad se emplean para definir e identificar el género en las letras de pop-rock y cómo se relacionan dichas marcas con la autenticidad que se construye en la puesta en escena de las canciones.

En este sentido, con el presente artículo se ha pretendido proporcionar un punto de partida e iluminar algunos aspectos para el análisis de la construcción lingüística y discursiva de uno de los rasgos estéticos fundamentales de los artistas de pop-rock más valorados: la autenticidad.

\section{Apéndice 1}

\begin{tabular}{|l|l|l|l|l|}
\hline & Artista & Género musical & Álbum/canción & Año $^{1}$ \\
\hline 1. & Lone Star & Pop & Mi calle & 1968 \\
\hline 2. & Paco Ibáñez & Cantautor & En el Olympia & 1969 \\
\hline 3. & Joan Manuel Serrat & Cantautor & Mediterráneo & 1971 \\
\hline 4. & Vainica Doble & Pop & Heliotropo & 1973 \\
\hline 5. & Solera & Folk-rock & Solera & 1973 \\
\hline 6. & Triana & Flamenco & El Patio & 1975 \\
\hline 7. & Veneno & Flamenco & Veneno & 1977 \\
\hline
\end{tabular}




\begin{tabular}{|c|c|c|c|c|}
\hline 8. & Moris & Rock & Fiebre de vivir & 1978 \\
\hline 9. & Burning & Rock & El fin de la década & 1979 \\
\hline 10. & Alaska y los Pegamoides & Movida & Grandes éxitos & 1982 \\
\hline 11. & Parálisis Permanente & Movida & El acto & 1982 \\
\hline 12. & Miguel Ríos & Rock & Rock y Ríos & 1982 \\
\hline 13. & Loquillo & Movida & El ritmo del garage & 1983 \\
\hline 14. & Radio Futura & Movida & La ley del desierto/La ley del mar & 1984 \\
\hline 15. & Gabinete Caligari & Movida & Cuatro rosas & 1984 \\
\hline 16. & Alaska y Dinarama & Movida & Deseo carnal & 1984 \\
\hline 17. & Golpes Bajos & Movida & A Santa Compaña & 1984 \\
\hline 18. & Miguel Bosé & Pop & Bandido & 1984 \\
\hline 19. & El Último de la Fila & Rock & Enemigos de lo ajeno & 1986 \\
\hline 20. & Hérores del silencio & Rock & Senderos de traición & 1990 \\
\hline 21. & Kiko Veneno & Flamenco & Échate un cantecito & 1992 \\
\hline 22. & Albert Pla & Cantautor & No sólo de rumba vive el hombre & 1992 \\
\hline 23. & Family & Indie & Un soplo en el corazón & 1993 \\
\hline 24. & Morente y Lagartija Nick & Flamenco & Omega & 1996 \\
\hline 25. & Extremoduro & Rock urbano & Agila & 1996 \\
\hline 26. & Alejandro Sanz & Pop & Más & 1997 \\
\hline 27. & Andrés Calamaro & Rock & Honestidad brutal & 1999 \\
\hline 28. & Joaquín Sabina & Cantautor & 19 días y 500 noches & 1999 \\
\hline 29. & Quique González & Rock & Avería y redención \#7 & 2007 \\
\hline
\end{tabular}




\section{Apéndice 2}

\begin{tabular}{|c|c|c|c|c|}
\hline & Artista & Categoría & $\begin{array}{l}\text { Álbum/canción } \\
\text { galardonado }\end{array}$ & Año ${ }^{2}$ \\
\hline 1. & Julio Iglesias & Best Latin Pop Performance & Un hombre solo & 1987 \\
\hline 2. & Luis Miguel & Pop Album & Amarte es un placer & 2000 \\
\hline 3. & Aterciopelados & Rock Duo/Group w/Vocal & Gozo poderoso & 2001 \\
\hline 4. & Christina Aguilera & Female Pop Vocal Album & Mi reflejo & 2001 \\
\hline 5. & Alejandra Guzmán & Best Rock Solo Vocal Album & Soy & 2002 \\
\hline 6. & Miguel Bosé & Best Male Pop Vocal Album & Sereno & 2002 \\
\hline 7. & Rosario & Mejor Álbum Vocal Pop Femenino & Muchas flores & 2002 \\
\hline 8. & Sin bandera & $\begin{array}{l}\text { Best Pop Album By A Duo Or } \\
\text { Group With Vocal }\end{array}$ & Sin bandera & 2002 \\
\hline 9. & Enrique Iglesias & Best Male Pop Vocal Album & Quizás & 2003 \\
\hline 10 & Juanes & Best Rock Solo Vocal Album & Un día normal & 2003 \\
\hline 11. & Olga Tañón & Mejor Álbum Vocal Pop Femenino & Sobrevivir & 2003 \\
\hline 12. & Alejandro Sanz & Best Male Pop Vocal Album & No es lo mismo & 2004 \\
\hline 13. & La Ley & $\begin{array}{l}\text { Best Rock Album By A Duo Or } \\
\text { Group With Vocal }\end{array}$ & Libertad & 2004 \\
\hline 14. & Marc Anthony & Best Latin Pop Album & Amar sin mentiras & 2004 \\
\hline 15. & Bacilos & $\begin{array}{l}\text { Best Pop Album By A Duo Or } \\
\text { Group With Vocal }\end{array}$ & Sinvergüenza & 2005 \\
\hline 16. & Gustavo Cerati & Best Rock Solo Vocal Album & Ahí vamos & 2006 \\
\hline 17. & Julieta Venegas & Best Latin Pop Album & Limón y sal & 2006 \\
\hline 18. & $\begin{array}{l}\text { La oreja de Van } \\
\text { Gogh }\end{array}$ & $\begin{array}{l}\text { Best Pop Album By A Duo Or } \\
\text { Group With Vocal }\end{array}$ & Guapa & 2006 \\
\hline 19. & Shakira & Mejor Álbum Vocal Pop Femenino & Fijación Oral Vol. 1 & 2006 \\
\hline 20. & Juan Luis Guerra & Grabación del año ${ }^{3}$ & La llave de mi corazón & 2007 \\
\hline
\end{tabular}




\begin{tabular}{|c|c|c|c|c|}
\hline 21. & Ricky Martin & Best Male Pop Vocal Album & $\begin{array}{l}\text { MTV Unplugged } \\
\text { (Sony BMG Music } \\
\text { Entertainment) }\end{array}$ & 2007 \\
\hline 22. & Andrés Calamaro & Best Rock Solo Vocal Album & La lengua popular & 2008 \\
\hline 23. & Belanova & $\begin{array}{l}\text { Best Pop Album By A Duo Or } \\
\text { Group With Vocal }\end{array}$ & Fantasía Pop & 2008 \\
\hline 24. & Kany García & Mejor Álbum Vocal Pop Femenino & Cualquier día & 2008 \\
\hline 25. & Jaguares & $\begin{array}{l}\text { Best Rock Album By A Duo Or } \\
\text { Group With Vocal }\end{array}$ & 45 & 2009 \\
\hline 26. & Laura Pausini & Mejor Álbum Vocal Pop Femenino & Primavera Anticipada & 2009 \\
\hline 27. & Camila & $\begin{array}{l}\text { Best Pop Album By A Duo Or } \\
\text { Group With Vocal }\end{array}$ & Dejarte de amar & 2010 \\
\hline 28. & Nelly Furtado & Mejor Álbum Vocal Pop Femenino & Mi plan & 2010 \\
\hline 29. & Alex, Jorge y Lena & $\begin{array}{l}\text { Best Pop Album By A Duo Or } \\
\text { Group With Vocal }\end{array}$ & Alex, Jorge y Lena & 2011 \\
\hline 30. & Maná & $\begin{array}{l}\text { Best Rock Performance By A Duo } \\
\text { Or Group With Vocal }\end{array}$ & Drama y luz & 2011 \\
\hline 31. & El cuarteto de Nos & Mejor álbum pop/rock & Porfiado & 2012 \\
\hline 32. & Jesse y Joy & $\begin{array}{l}\text { Mejor álbum vocal pop contem- } \\
\text { poráneo }\end{array}$ & $\begin{array}{l}\text { ¿Con quién se queda el } \\
\text { perro? }\end{array}$ & 2012 \\
\hline 33. & Beto Cuevas & Mejor álbum pop/rock & Transformación & 2013 \\
\hline 34. & Jorge Drexler & Grabación del año & Bailar en la cueva & 2014 \\
\hline 35. & Vicentico & Mejor canción de rock & Último acto & 2015 \\
\hline
\end{tabular}

\section{Notas}

1 Referido al año en que se editó cada álbum y/o canción.

2 Referido al año en que resultó premiado cada artista. Fuente: http://www.latingrammy.com/es/ nominees (consulta 10.06.2016).

3 Los dos artistas del corpus que fueron galardonados en la categoría "Grabación del año" (Juan Luis Guerra en 2007 y Jorge Drexler en 2014) son considerados “cantautores" en la Tabla 3 (Composición del corpus según subgéneros musicales). 


\section{Bibliografía}

Appen, Ralph: “Schein oder nicht-Schein? Zur Inszenierung von Authentizität auf der Bühne”. En: Helms, Dietrich / Phleps, Thomas (eds.): Ware Inszenierungen. Performance, Vermarktung und Authentizität in der populären Musik. Bielefeld: transcript, 2013, 41-70.

Appen, Ralph / Doehring, André: “Nevermind the Beatles. Here’s Exile 61 and Nico: ,The Top 100 Records of All Time': A Canon of Pop and Rock Albums from a Sociological and an Aesthetic Perspective”. En: Popular Music, 25/1 (2006), 21-39.

Auslander, Philip: "Musical Personae". En: The Drama Review 50/1 (2006), 100-119.

Bértoli-Dutra, Patrícia: "Multi-Dimensional analysis of pop songs". En: Berber Sardinha, Tony I Veirano Pinto, Marcia (eds.): Multi-Dimensional Analysis, 25 years on. A tribute to Douglas Biber. Amsterdam/Philadelphia: John Benjamins Publishing Company, 2016, 149-176.

Böhm, Thomas: "Give me some truth. Vorläufige Überlegungen zur Konstruktion von Authentizität in der Musik”. En: Rösing, Helmut / Phleps, Thomas (eds.): Populäre Musik im kulturwissenschaftlichen Diskurs. Karben: CODA, 2000, 251-262.

Brackett, Donald: Dark Mirror. The Pathology of the Singer-Songwriter. Westport: Praeger, 2008.

Bubenhofer, Noah: Sprachgebrauchsmuster. Korpuslinguistik als Methode der Diskurs- und Kulturanalyse. Berlin/New York: Walter de Gruyter, 2009.

Eckstein, Lars: Reading Song Lyrics. Amsterdam/New York: Rodopi, 2010.

Fouce, Héctor: "Nadie puede parar. La industria del rock en España, 1975-1985”. En: Kiko Mora / Eduardo Viñuela (eds.): Rock around Spain. Historia, industria, escenas y medios de comunicación. Lleida: espai / temps, 2013.

Frith, Simon: "Why do songs have words?" En: Contemporary Music Review 5 (1989), 77-96.

Frith, Simon: Performing Rites. On the Value of Popular Music. Cambridge (M): Harvard University Press, 1996.

Halliday, Michael A.K.: Language as social semiotic. London: Edward Arnold, 1978.

Jaedtke, Wolfgang: "Popmusik als Epochenstil. Versuch einer musikhistorischen und musiktheoretischen Aufarbeitung”. En: Rösing, Helmut / Phleps, Thomas (eds.): Populäre Musik im kulturwissenschaftlichen Diskurs. Karben: CODA, 2000, 201-216.

Kreyer, Ralph y Mukherjee, Joybrato: “The style of pop song lyrics: A corpus-linguistic pilot study”. En: Anglia 125/1 (2007), 31-58.

Laferl, Christopher: "Record it, and let it be known". Song Lyrics, Gender and Ethnicity from 1920 to 1960. Wien: LIT, 2005.

Moore, Allan: "Authenticity as Authentication”. En: Popular Music 21/2 (2002), 209-223.

Murphey, Tim: Song and Music in Language Learning. An Analysis of Pop Song Lyrics and the Use of Song and Music in Teaching English to Speakers of other Languages. Frankfurt am Main: Peter Lang, 1990.

Noya, Javier y Pérez-Colman, Martín: “¿Potencia sonora? España en los premios Grammy”. En: http:/www.realinstitutoelcano.org/wps/portal/web/rielcano_es/contenido?WCM_GLOBAL_ CONTEXT=/elcano/elcano_es/zonas_es/dt12-2011 (consulta 10.06.2016). 
Val, Fernán del / Noya, Javier / Pérez-Colman, Martín: “¿Autonomía, sumisión o hibridación sonora? La construcción del canon estético del pop-rock español”. En: Revista española de investigaciones sociológicas 145 (2014), 147-180.

Werner, Valentin: "Love is all around: a corpus-based study of pop-lyrics". En: Corpora 7/1 (2012), $19-50$. 